\title{
Tuberculosis in the Central Nervous System
}

\section{Guerrero-De León JE ${ }^{1}$ and Rodríguez-Leyva $\mathbf{I}^{1,2 *}$}

${ }^{1}$ School of Medicine, Universidad Autónoma De San Luis Potosí

${ }^{2}$ Neurology Department, Hospital Central "Dr. Ignacio Morones Prieto". San Luis

Potosí, S.L.P., México

*Corresponding Author: Rodríguez-Leyva I, School of Medicine, Universidad

Autónoma De San Luis Potosí and Neurology Department, Hospital Central

“Dr. Ignacio Morones Prieto". San Luis Potosí, S.L.P., México

DOI: 10.31080/ASMI.2020.03.0513
Received: January 03, 2020

Published: February 08, 2020

(C) All rights are reserved by Guerrero-De

León JE and Rodríguez-Leyva I.

\begin{abstract}
Tuberculosis (TB) is an ancient, reemerging, infectious, chronic and highly fatal disease. It is caused by bacilli of the genus $M y-$ cobacterium and is considered as a complex of microbiological and immunological phenomena that represents a significant public health issue in Mexico and in the world [1]. According to the World Health Organization, it is estimated that a quarter of the world's population has latent TB, that is, they are infected by the bacilli, but have not yet become ill or can not spread the infection [2]. These individuals have a $5-15 \%$ risk of developing the illness throughout their lives, and two million will die because of this disease [3].

Keywords: Tuberculosis Central Nervous System; Tuberculosis Meningeal; Cerebral Ventriculitis; Tuberculoma; Arachnoiditis; Drug-Resistant Tuberculosis
\end{abstract}

\section{Abbreviations}

Tb: Tuberculosis; SNC: Central Nervous System; TBM: Tuberculous Meningitis; CSF: Cerebrospinal Fluid; SNC-Tb: Central Nervous System Tuberculosis

\section{Introduction}

The Mycobacterium genus comprises over 50 species, of which Mycobacterium tuberculosis and Mycobacterium bovis stand out. The first is transmitted by inhalation of infectious material, while the latter, through the ingestion of contaminated cow's milk [4].

These microorganisms are characterized by being difficult to stain, manifesting their acid resistance. In addition, they are obligated aerobes, without motility and do not create spores. The cell wall is composed of peptidoglycan, mycolic acids (which is why they are called "mycobacteria") and lipoarabinomannan (LAM). This composition causes the cell wall to have a high lipid content that would justify its biological peculiarities. Because of acid resistance, the most commonly utilized microbiological procedure to diagnose TB in the world is microscopy with Ziehl-Neelsen or Kinyoun staining. Its antigenic composition consists of different proteins, including tuberculin as the most studied antigen $[5,6]$.

\section{Outlook of tuberculosis in Mexico}

The official data in 2016 of the Single Information System for Epidemiological Surveillance in Mexico revealed 16,913 new cases of pulmonary TB, with a morbidity rate of 13.8 per 100,000 population. Regarding meningeal TB, 373 new cases were found in the same year, and their morbidity rate was 0.3 per 100,000 population. In our State of San Luis Potosí, 6 new cases of meningeal TB, as well as a morbidity rate of 0.2 per 100,000 population were recorded [7].

Pathogenesis of tuberculosis

Most TB infections develop because of the inhalation of droplet nuclei that carry the microorganism. Besides this, people can become infected through the gastrointestinal tract (by ingestion 
of cow's milk contaminated with M. bovis) or by skin abrasions. Because a simple cough access can generate up to 3000 infected droplet nuclei -and given the fact that hardly fewer than 10 bacilli are needed to initiate a lung infection-, the probability of acquiring the infection is directly associated to the amount of microorganisms present in the sputum and with the frequency of cough accesses in the patient [8].

\section{Primary infection}

It comprises the deposition of the tuberculous bacilli in the peripheral alveoli, particularly in the middle and lower lobe because of better ventilation. After this, and because it is an intracellular pathogen, the bacillus spreads within the alveolar macrophages and these cells are transported to the hilar lymph nodes, causing a low-level bacteraemia that spreads the bacillus to different tissues, including the central nervous system (SNC). The body's response to this situation is the development of a granuloma, formed by the mixture of delayed hypersensitivity responses, and the TH1-type immune response. This granuloma contains the bacilli, deprives them of oxygen and limits their intracellular growth [9].

\section{HIV and tuberculosis}

It is evidently that the disturbance of the immune system in patients with human immunodeficiency virus (HIV) infection is a predisposing factor for developing TB, with a $30 \%$ risk in the first year of diagnosis (compared with 3\% risk for the general population) $[10,11]$.

\section{Pathogenesis of tuberculosis in the central nervous system}

In the CNS, the disease appears with the development of small tuberculous foci (known as "Rich foci") in the brain, in the meninges or in the spinal cord. The location of these foci, and the individual's ability to handle them, will determine how TB occurs: frequently it will present as tuberculous meningitis (TBM) and, to a slighter degree, as encephalitis, intracranial tuberculoma or as a brain abscess. Tuberculous meningitis develops more regularly as a complication of a primary infection in young children and infants. In immunocompromised adults, it will develop due to chronic reactivation of bacillemia, promoted by age, alcoholism, malnutrition, malignancy, HIV infection or by intake of drugs that suppress Tumor Necrosis Factor- $\alpha$ (TNF- $\alpha$ ). In addition to this, head trauma can lead to the destabilization of a previously quiescent foci, conditioning meningitis in the absence of a generalized infection $[12,13]$.
TNF- $\alpha$ plays a definitive role in granuloma formation and in the containment of mycobacterial infection. The production of this cytokine in the CNS contributes to the disruption of the blood-brain barrier and the presence of leukocytosis in the cerebrospinal fluid (CSF) [12]. CNS macrophages (the microglia cells) are target of mycobacterias, leading to increased production of cytokines and chemokines (including TNF- $\alpha$ and interleukin 6, among others) [14].

Once the bacilli are released in the subarachnoid space, a thick gelatinous exudate rich in erythrocytes, neutrophils and macrophages will form mostly in the interpeduncular fossa and in the anterior suprasellar region, which can extend to the prepontine cistern and surround the entire spinal cord. This exudate can involve cranial nerves and arteries, conditioning a "bottleneck" effect on the flow of CSF at the level of the tentorial opening, and developing hydrocephalus. In addition, the exudate generates damage to the underlying brain parenchyma. One of the most severe complications of TBM is the development of vasculitis in the circle of Willis, in the vertebrobasilar system and in the perforating branches of the middle cerebral artery, that may precipitate infarctions throughout the distribution of these vessels [12,13].

Tuberculomas develop in the cerebral parenchyma due to the enlargement and disruption of a tuberculous foci. They can appear alongside TBM or separately. Pathologically, tuberculomas are characterized by granulomatous reaction with epithelioid cells and giant cells mixed with lymphocytes that surround an area of caseous necrosis [15].

\section{Clinical features of the central nervous system tuberculosis}

TBM corresponds to $1 \%$ of all cases of $\mathrm{TB}$ and represents $5 \%$ of all cases of extrapulmonary disease in immunocompetent patients [16]. It is vital to recognize this entity as soon as possible because the clinical outcome depends mostly on the stage in which the treatment is initiated. Because of this, antituberculous drugs should be initiated immediately in any patient with meningeal syndrome accompanied by data of hypoglycorrhachia, hyperproteinorrachia, and lymphocytic pleocytosis or with evidence of TB in any region of the body [17].

The clinical manifestations of TBM can be divided into three distinguishable phases [18]:

- $\quad$ Prodromal phase: Duration of 2-3 weeks. Characterized by having an insidious onset followed by malaise, headache, fatigue, fever and personality changes. 
- Meningeal phase: Characterized by frank neurological alterations such as meningism, headache and vomiting, lethargy, confusion and various degrees of cranial nerves and long tracts involvement.

- Paralytic phase: Begins as the disease progresses faster. It is characterized by stupor, coma, epileptic seizures and hemiparesis. In patients who do not receive adequate treatment, this phase can condition death within 5 - 8 weeks after the onset of the disease.

Almost 30\% of patients with TBM have underlying miliary TB, so the finding of choroidal granulomas in ophthalmoscopy is not surprising [19].

Tuberculomas in the brain parenchyma can increase their size substantially and not condition meningeal inflammation (known as silent tuberculomas). However, symptomatic tuberculomas are characterized by causing a picture of epileptic seizures or headache, sometimes accompanied by hemiplegia and signs of increased intracranial pressure. They are commonly accompanied by symptoms of systemic disease or meningeal inflammation. Its main differential diagnosis is with a subacute or chronic meningeal syndrome, characterized by CSF with lymphocyte pleocytosis, hypoglycorrhachia and hyperproteinorrachia, such as the one observed in Cryptococcus, and Brucella infection and in patients with neurosyphilis [18,20].

Other forms of presentation of tuberculosis in the central nervous system

Besides TBM, CNS-TB can occur in the form of ventriculitis, abscess, vasculitis with secondary infarctions, arachnoiditis and vertebral tuberculosis (Pott's disease). These will be concisely addressed in the following paragraphs.

\section{Ventriculitis}

It is one of the rarest and most devastating complications of CNS-TB. This occurs after the enlargement, extension and disruption of a foci located in the ventricular system. This enlargement is observed in $30 \%$ of patients receiving antituberculous treatment as a paradoxical sign of inflammatory syndrome of immune reconstitution associated with TB, and a mortality of $13 \%$ has been reported [21,22].

\section{Tuberculous brain abscess}

It is a rare manifestation of CNS-TB that is generally comparable to pyogenic brain abscess more than with tuberculomas. The abscess is generally larger than a tuberculoma and is characterized by cavitation with pus in its center [23]. Its definitive diagnosis is established with microscopy and with culture of the purulent material [31].

\section{Vasculitis}

In a study by Dastur., et al. [24] ischemic stroke data generated by vasculitis were found in $41 \%$ of a set of 100 autopsies of patients diagnosed with TBM.

Cerebral vasculitis owing to TB is a complication of TBM, with an estimated incidence of 1.5 - 5\%. As a result, narrowness of the middle cerebral arteries, anterior cerebral arteries and internal carotid arteries is frequently found. Javaud., et al. [25] determined an average time of onset of 40 days (ranging from 0-139 days) after the beginning of antituberculous therapy and despite the use of corticosteroids [25].

This entity should be included in the differential diagnosis of any neurological impairment that arises during the course of TB, most importantly of TBM [26].

\section{Arachnoiditis}

TB infection in the spinal cord develops in different ways, such as spondylitis, intradural tuberculosis and trabecular myelitis. "Spinal arachnoiditis" is another term to refer to intradural tuberculosis. It has been agreed that these forms of atypical presentation of TB should be considered as tuberculous radiculomelopathy (TBRM), which has three stages:

- Radiculitis: Inflammation of the arachnoid with hyperemia and edema of the nerve roots.

- Arachnoiditis: Proliferation of fibroblasts and collagen deposition that determines the entrapment of nerve roots towards the pia arachnoid.

- $\quad$ Adhesive arachnoiditis: Dense collagen deposition with nerve encapsulation $[27,28]$.

Vertebral tuberculosis

Osteoarticular involvement of TB is particularly frequent, but its diagnosis becomes complex in clinical practice. Although it usually involves any bone, the spine is the most frequent site affected. Also recognized as "Pott's disease," it is a form of extrapulmonary TB that preferentially involves the T8 - L3 vertebrae. It has its origin 
in a primary pulmonary focus (exceptionally urogenital), which facilitates a secondary foci at the bone level, disseminating hematogenously or through the prevertebral lymphatics. The lesion causes progressive vertebral destruction that provokes continuous pain, local stiffness and stiffness, spasm of the paraspinal muscles, and spinal deformity in the patient. Its has a slow and insidious progression, with an average duration of 4 - 11 months. It is usually accompanied by neurological deficits if the thoracic and cervical region is involved. Without treatment, it can condition paraplegia or tetraplegia $[29,30]$.

\section{Diagnosis of tuberculosis in the central nervous system}

Being able to diagnose CNS-TB demands the combined analysis of the interrogation, clinical findings, and data from neuroimaging studies and from laboratory studies (including CSF analysis) 31.

\section{Cerebrospinal fluid}

Typically, a lymphocytic pleocytosis will be identified with an average of 200 cells/ $\mu \mathrm{l}$, moderate-severe hyperproteinorrachia $(0.5-3 \mathrm{~g} / \mathrm{L})$ and hypoglycorrhachia less than $45 \mathrm{mg} / \mathrm{dL}$ or less than $40-50 \%$ of the serum glucose concentration. Likewise, lactate levels may be increased ( $5-10 \mathrm{mmol} / \mathrm{L}$ ) and the determination of IgG antibodies can help differentiate this pathology from aseptic meningitis (100\% sensitivity and $83 \%$ specificity) [31,32].

It is fundamental to perform culture for mycobacteria. For this, the Löwenstein-Jensen medium is employed, an average development time of $12-24 \mathrm{~h}$ is needed, and a specimen with at least 10,000 colony-forming units is necessary to confirm the diagnosis, although the results are positive only in the $5-58 \%$ of cases $[5,23]$. As previously mentioned, the tuberculous bacillus in the CSF can be identified through Ziehl - Neelsen or Kinyoun staining [5,34].

\section{Neuroimaging studies}

Computed tomography (CT) and magnetic resonance imaging (MRI) are of exceptional interest when accompanied by clinical manifestations that enhance the diagnostic impression. It is crucial to perform them in a way that encompasses the entire neuroaxis $[33,35]$.

MRI with contrast is considered the method-of-choice for the detection of CNS-TB, as it provides stronger diagnostic information. In these images a triad can be found, characterized by 1) basal meningeal reinforcement and basal exudates; 2) hydrocephalus and 3) regions with infarct data [25]. Tuberculomas are the $4^{\text {th }}$ most constant finding. These manifestations can appear in isolation or simultaneously, however, most of the radiographic findings per se lack sufficient sensitivity. Only meningeal reinforcement has a sensitivity of $89 \%$ [36].

\section{Nucleic acid studies}

If available, CSF nucleic acid amplification studies should be performed, especially in patients with significant clinical suspicion and with negative results in the other diagnostic tools. The World Health Organization suggested in 2017 the use of Xpert MTB/RIF Ultra assay as an initial diagnostic test for patients with tuberculous meningitis [37].

\section{Treatment of tuberculosis in the central nervous system}

Without treatment, CNS-TB can lead to the death of the patient. Therefore, the effective treatment must eradicate the active bacilli to prevent neurological sequelae, and it must also get rid of the quiescent bacilli to prevent recurrences and the generation of antimicrobial resistance [38].

Unlike pulmonary TB, TBM does not have international standardized recommendations for its management. In part, this may be because of the reduced evidence that deals with the proper regime of antituberculous drugs, as well as its most appropriate duration. Most of these data are retrieved by extrapolating the management of pulmonary TB $[39,40]$.

The management of TB in Mexico is comparable to the international standardized treatment. It begins with an intensive regimen consisting of four drugs and lasting two months, after which it proceeds with a prolonged support regime consisting of two drugs only and lasting another 7 - 10 months (Table 1) [41,42].

In patients in whom we do not suspect drug resistance, the intensive regimen is administered orally for two months and includes isoniazid, rifampicin, pyrazinamide and ethambutol. Once finished, the prolonged support regime is established orally for 7 - 10 months, and includes only isoniazid and rifampicin. In patients with the presence of tuberculoma, we should maintain the treatment for 18 months [41,42]. 


\begin{tabular}{|l|c|c|}
\hline Intensive Regimen & $\begin{array}{c}\text { Daily, From Monday To Saturday, Until 60 Doses } \\
\text { Are Completed. Administration In One Shot }\end{array}$ & Adverse Effects \\
\hline Rifampin (R) & $600 \mathrm{mg}$ & $\begin{array}{c}\text { Hepatitis, febrile reaction, } \\
\text { purpura, hypersensitivity, } \\
\text { oral intolerance }\end{array}$ \\
\hline Isoniazide (H) & $300 \mathrm{mg}$ & $\begin{array}{c}\text { Neuritis, hepatitis, hypersen- } \\
\text { sitivity, lupoid syndrome }\end{array}$ \\
\hline Pyrazinamide (Z) & $1500-2000 \mathrm{mg}$ & $\begin{array}{c}\text { Gouty arthropathy, hyperuri- } \\
\text { cemia, hepatitis, gastritis }\end{array}$ \\
\hline Ethambutol (E) & $1200 \mathrm{mg}$ & $\begin{array}{c}\text { Optic neuritis, red-green } \\
\text { discrimination }\end{array}$ \\
\hline $\begin{array}{l}\text { Prolonged } \\
\text { support regime }\end{array}$ & $\begin{array}{c}\text { Wednesday and Friday), until 45 doses are } \\
\text { completed. Administration in one shot }\end{array}$ & $\begin{array}{c}\text { Adverse Effects } \\
\text { Isoniazide (H) } \\
\text { Rifampin (R) }\end{array}$ \\
\hline
\end{tabular}

Table 1: Primary tuberculosis treatment in Mexico [42].

Isoniazid, rifampin and pyrazinamide are bactericidal drugs that can be administered orally and that adequately penetrate inflamed meninges, so they reach CSF concentrations above their inhibitory concentration necessary to act on susceptible strains [43].

In addition, it is recommended to administer glucocorticoids simultaneously, as there is evidence that these reduce the mortality rate (RR of 0.7 , with a $95 \%$ confidence interval of $0.65-0.87$ ). Although the regimen remains undetermined, it is recommended to use Dexamethasone in the following schedule:

- $\quad$ First 2 weeks: $0.3-0.4 \mathrm{mg} / \mathrm{Kg} / \mathrm{d}$ intravenously.

- $\quad 3^{\text {rd }}$ week: $0.2 \mathrm{mg} / \mathrm{Kg} / \mathrm{d}$ intravenously.

- $\quad 4^{\text {th }}$ week: $0.1 \mathrm{mg} / \mathrm{Kg} / \mathrm{d}$ intravenously

- $\quad 5^{\text {th }}$ week: $4 \mathrm{mg} / \mathrm{d}$ orally, decreasing $1 \mathrm{mg}$ per week until completing 8 weeks of treatment [44].

A particular situation in relation to the management of TBM appears in pediatric patients, as the American Academy of Pediatrics suggests replacing ethambutol with ethionamide or with an aminoglycoside (like streptomycin), because this drug is related with optic neuritis symptoms [45].

\section{Bibliography}

1. World Health Organization. Global tuberculosis report (2019).

2. World Health Organization. Tuberculosis fact sheet (2018).
3. Kurt T. Tuberculosis, detección de casos, tratamiento y vigilancia. Organización Panamericana de la Salud. Segunda edición. Washington, D.C (2004).

4. Riley LW. Tuberculosis: Natural history, microbiology, and pathogenesis (2019).

5. McNeil MR and Brennan PJ. "Structure, function and biogenesis of the cell envelope of mycobacteria in relation to bacterial physiology, pathogenesis and drug resistance some thoughts and possibilities arising from recent structural information". Research in Microbiology 142.4 (1991): 451.

6. Allen BW and Mitchison DA. "Counts of viable tubercle bacilli in sputum related to smear and culture gradings". Medical Laboratory Scientist 49.2 (1992): 94.

7. Centro Nacional de Programas Preventivos y Control de Enfermedades (CENAPRECE). Programa preventivo de dirección de micobacteriosis.

8. Rom WN and Garay SM. "Tuberculosis". $2^{\text {nd }}$ ed. Philadelphia, PA: Lippincott Williams and Wilkins (2004).

9. Ryan KJ. "Sherris Medical Microbiology". $7^{\text {th }}$ Edition. United States: McGraw-Hill Education (2018).

10. Shafer RW. "Extrapulmonary tuberculosis in patients with human immunodeficiency virus infection". Medicine (Baltimore) 70.6 (1991): 384-397. 
11. Zumla A., et al. "Impact of HIV infection on tuberculosis". Postgraduate Medical Journal 76 (2000): 259-268.

12. Rock RB., et al. "Central Nervous System Tuberculosis: Pathogenesis and Clinical Aspects". Clinical Microbiology Reviews 21.2 (2008): 243-261.

13. Dastur DK., et al. "Pathology and pathogenetic mechanisms in neurotuberculosis". Radiologic Clinics of North America 33.4 (1995): 733.

14. Rock RB., et al. "Role of microglia in central nervous system infections”. Clinical Microbiology Reviews 17.4 (2004): 942-964.

15. Rich AR and Mc Cordock HA. "The pathogenesis of tuberculous meningitis". Bulletin of the Johns Hopkins Hospital 52 (1933): 5-37.

16. Centers for Disease Control and Prevention. Reported tuberculosis in the United States, 2013. US Department of Health and Human Services, Atlanta, GA (2014).

17. Navarro-Cano G., et al. "Tuberculosis (TB) del sistema nervioso central (SNC). Experiencia de 3 años en su diagnóstico y tratamiento en el Hospital Central Dr. Ignacio Morones Prieto de San Luis Potosí, S.L.P”. Revista Mexicana de Neurociencia 1.3 (2000): 12-18.

18. Leonard JM. “Central nervous system tuberculosis". Bernardo J, Edwards MS (2019).

19. Kennedy DH and Fallon RJ. "Tuberculous meningitis". Journal of the American Medical Association. 241.3 (1979): 264.

20. Kumar R., et al. "Tuberculous brain abscess: clinical presentation, pathophysiology and treatment (in children)". Child's Nervous System 18 (2002): 118-123.

21. Vaziri S., et al. "Tuberculous ventriculitis: A rare complication of central nervous system tuberculosis". International Journal of Mycobacteriology 5 (2016): 231-234.

22. Pepper DJ., et al. "Neurologic Manifestations of Paradoxical Tuberculosis-Associated Immune Reconstitution Inflammatory Syndrome: A Case Series". Clinical Infectious Diseases 48.11 (2009): e96-e107.

23. Chakraborti S., et al. "Clinicopathological study of tuberculous brain abscess”. Pathology - Research and Practice 205 (2009): 815-822.

24. Dastur DK., et al. "The brain and meninges in tuberculous meningitis. Gross pathology in 100 cases and pathogenesis". Neurology 18 (1970): 86-100.
25. Javaud N., et al. "Tuberculous cerebral vasculitis: retrospective study of 10 cases". European Journal of Internal Medicine 22.6 (2011): e99-104.

26. Sharon-Tai ML., et al. "Cerebral infarction pattern in tuberculous meningitis". NATURE Scientific Reports 6.38802 (2016): $1-10$.

27. Konar SK., et al. "Tuberculous lumbar arachnoiditis mimicking conus cauda tumor: A case report and review of literature". Journal of Neurosciences in Rural Practices 2.1 (2011): 93-96.

28. Hoffman GS. "Spinal arachnoiditis: What is the clinical spectrum?". Spine 8 (1983): 538-540.

29. Arias-Deroncerés IJ., et al. "Tuberculosis vertebral (mal de Pott) e infección por el virus de la inmunodeficiencia humana". Medisan 15.12 (2011): 1791-1797.

30. Kumar Garg R and Singh Somvanshi DS. "Spinal tuberculosis: A review". The Journal of Spinal Cord Medicine 34.5 (2011): 440454.

31. Schaller MA., et al. "Central Nervous System Tuberculosis: Etiology, Clinical Manifestations and Neuroradiological Features". Clinical Neuroradiology 29.1 (2019): 3-18.

32. Garcia-Monco JC. “Tuberculosis". In: Biller J, Ferro J, editors. Handb Clin Neurol. Neurol Asp Syst Dis Part III. 3rd Series 121. Amsterdam: Elsevier (2014).

33. Thwaites G., et al. "British Infection Society guidelines for the diagnosis and treatment of tuberculosis of the central nervous system in adults and children". Journal of Infection 59 (2009): 167-187.

34. Jeren $\mathrm{T}$ and Beus I. "Characteristics of cerebrospinal fluid in tuberculous meningitis". Acta Cytologica 26 (1982): 678-680.

35. Bernaerts A., et al. "Tuberculosis of the central nervous system: overview of neuroradiological findings". European Radiology 13 (2003): 1876-1890.

36. Botha H., et al. "Reliability and diagnostic performance of CT imaging criteria in the diagnosis of tuberculous meningitis". PLoS One 7 (2012): e38982.

37. WHO. Meeting Report of a Technical Expert Consultation: Noninferiority analysis of Xpert MTB/RIF Ultra compared to Xpert MTB/RIF (2017).

38. Woodfield J and Argent A. "Evidence behind the WHO guidelines: hospital care for children: what is the most appropriate anti-microbial treatment for tuberculous meningitis?". Journal of Tropical Pediatrics 54.4 (2008): 220-224. 
39. Jullien S., et al. "Six months therapy for tuberculous meningitis (Review)". Cochrane Database of Systematic Reviews 9 (2016): 012091.

40. Heemskerk D., et al. "Intensified treatment with high dose rifampicin and levofloxacin compared to standard treatment for adult patients with tuberculous meningitis (TBM-IT): protocol for a randomized controlled trial". Trials 12 (2011): 25.

41. Nahid P., et al. "Official American Thoracic Society/Centers for Disease Control and Prevention/Infectious Diseases Society of American clinical practice guidelines: Treatment of drug-susceptible tuberculosis". Clinical Infectious Diseases 63 (2016): e147.

42. Para la prevención y control de la tuberculosis. Norma Oficial Mexicana NOM-006-SSA2-2013. Diario Oficial de la federación (2013).

43. Donald PR. "Cerebrospinal fluid concentrations of antituberculosis agents in adults and children". Tuberculosis 90.5 (2010): 279.

44. Thwaites GE., et al. "Dexamethasone for the treatment of tuberculous meningitis in adolescents and adults". The New England Journal of Medicine 351.17 (2004): 1741.

45. American Academy of Pediatrics. Red Book:Report of the Committee on Infectious Diseases, 31st ed, Kimberlin DW, Brady MT, Jackson MA, Long SS (Eds), American Academy of Pediatrics, Itasca, IL (2018).

\section{Assets from publication with us}

- Prompt Acknowledgement after receiving the article

- Thorough Double blinded peer review

- Rapid Publication

- Issue of Publication Certificate

- High visibility of your Published work

Website: https://www.actascientific.com/

Submit Article: https://www.actascientific.com/submission.php

Email us: editor@actascientific.com

Contact us: +919182824667

Citation: Guerrero-De León JE and Rodríguez-Leyva I. “Tuberculosis in the Central Nervous System". Acta Scientific Microbiology 3.3 (2020): 01-07. 